\title{
The role of nutrition in patients with fibromyalgia: Is there an impact on disease parameters?
}

\author{
(1) Özlem Köroğlu¹, (D Kübra Tel Adıgüzel²
}

1 University of Health Sciences Turkey, Gülhane Faculty of Medicine; Gaziler Physical Medicine and Rehabilitation Training and Research Hospital, Ankara, Turkey

2University of Health Sciences Turkey, Gülhane Faculty of Health Sciences, Department of Nutrition and Dietetics, Ankara, Turkey

\section{Date submitted:}

11.04.2020

Date accepted:

05.06.2020

Online publication date:

15.09.2020

\section{Corresponding Author:}

Kübra Tel Adıgüzel, PhD, Asist Prof, University of Health Sciences Turkey, Gülhane Faculty of Health Sciences, Department of Nutrition and Dietetics, Ankara, Turkey

dyt.kubra@gmail.com

ORCID:

orcid.org/0000-0003-1010-4316

Keywords: Fibromyalgia, nutrition, biochemical parameters

\begin{abstract}
Aims: The aim of our study is to assess the associations between dietary intake and biochemical parameters, clinical assessments of pain, sleep quality and current health status of patients who were diagnosed with fibromyalgia.

Methods: This is a prospective study on patients with fibromyalgia. In patients' routine controls, data were recorded about their nutrition by using food consumption recording form. Also, the biochemical parameters were recorded. Clinical symptoms were evaluated by using Short form of MacGill Pain Questionnaire (SFMPQ), Fibromyalgia Impact Questionnaire (FIQ) and Pittsburg Sleep Quality Index (PSQI).

Results: Eighty-one patients with a disease duration of minimum 12 months were included. The mean age was 48.9 years and $90.1 \%$ of patients were female. The correlation analysis between serum vitamin $B_{12}$ and clinical symptoms showed a weak negative correlation with FIQ ( $r=-$ $0.257, p=0.021)$, sleep duration $(r=-0.317, p=0.004)$, sleep disturbance $(r=-0.279, p=0.012)$, sleep quality $(r=-0.235, p=0.035)$, and PSQI total score $(r=-0.316, p=0.004)$. Percentage of energy from dietary carbohydrate showed a moderate positive correlation with FIQ $(r=0.383, p<0.001)$. Dietary fat showed a moderate negative correlation with FIQ $(r=-0.29, p=0.007)$. While dietary saturated fat showed a moderate positive correlation with FIQ $(r=0.361, p<0.001)$, dietary unsaturated fat displayed a weak negative correlation with FIQ $(r=-0.228, p=0.041)$. Dietary fiber had a moderate negative correlation with FIQ $(r=-0.357, p<0.001)$, SFMPQ-present pain intensity $(r=-0.357, p<0.001)$, and SFMPQ-visual analogue scale $(r=-0.419, p<0.001)$ subscores.
\end{abstract}

Conclusions: The current study results suggest that a healthy diet habit consisting of low saturated fat, carbohydrates and higher fiber, vitamins and minerals may provide improvement in fibromyalgia symptoms. Dietary assessment and intervention should be a part of the management of patients with fibromyalgia.

\section{Introduction}

Fibromyalgia is a chronic disease characterized by general pain in the body with particular tender points, fatigue, depression, anxiety, non-restorative sleep, morning stiffness, headaches, cognitive impairment, paresthesia, affective disorders, irritable bowel and bladder syndrome. Fibromyalgia symptoms can be associated with several factors such as dysfunction of hypothalamic axis and increase of cortisol, changes in the central nervous system, oxidative stress, mitochondrial dysfunction, and also changes in the intestinal microbiota $(1,2)$. As the etiology is multifactorial, a multidimensional management which consists of a combination of pharmacological and non-pharmacological treatments is necessary for fibromyalgia'. Nutrients and nutritional supplements are also effective factors for fibromyalgia patients $(3,4)$. There is a linkage between dietary patterns and fibromyalgia symptoms $(5,6)$. However, according to the literature, the effects of different dietary approaches are not clear yet. A low fermentable oligo, di and monosaccharides, alcohols and polyols (FODMAPS) diet, a raw vegetarian diet and a hypocaloric diet may relieve pain and improve functional parameters in fibromyalgia patients. A 
nutritionally balanced diet contributing to weight loss can reduce the severity of fibromyalgia symptoms (7).

Fibromyalgia is a chronic disease caused by some neurologic, genetic, psychological conditions or mineral-vitamin deficient status. However, to date, there are few studies linking biochemical parameters and fibromyalgia. A deficit of nutritive elements including necessary metal ions and vitamins might have an important effect in the occurrence of fibromyalgia. Patients with deficiencies of some fundamental nutrients may develop dysfunction of pain pathways together with fibromyalgia symptoms (8).

In the treatment of fibromyalgia, some minerals and vitamins have beneficial effects. Sufficient intake of vitamin $D$, vitamin $B_{12}$ and magnesium is important. Deficiency of certain essential elements may cause dysfunction of pain inhibitory mechanisms. Insufficient levels of magnesium and selenium cause muscle pains. Deficiency of magnesium can cause chronic systemic inflammation, increase substance $P$ levels and induce an increase in pro-inflammatory cytokines and C-reactive protein (CRP). Selenium has a protective role against ischemia (8).

Iron is necessary for enzymes in neurotransmitter production. Investigation of cerebrospinal fluid in fibromyalgia syndrome has shown a decrease in the concentration of serotonin, dopamine and norepinephrine (8). Moreover, as iron is a cofactor in dopamine and serotonin synthesis, it may have an effect in the etiopathogenesis of fibromyalgia $(8,9)$. Therefore, the management of diet is very important for fibromyalgia patients in order to ameliorate inadequate intake of essential nutrients. When optimal nutrition is obtained, pain levels are usually decreased. Also, by consuming antioxidantrich foods, it is possible to relieve fibromyalgia symptoms.

Researches about nutrition are necessary to reveal relationships between nutrition and fibromyalgia. The aim of our study is to assess the associations between dietary intake and biochemical parameters, clinical assessments of pain, sleep quality and current health status of patients who were diagnosed with fibromyalgia.

\section{Methods}

This was a prospective study conducted between April 2019 and September 2019 on patients with fibromyalgia (diagnosed with American College of Rheumatology 2010 Criteria), who were admitted to the physical medicine and rehabilitation clinics. Eighty-one patients with a disease duration of minimum 12 months aged between 18 and 65 years were included. The patients were selected according to the use of similar medical treatment and having similar exercise habits. Exclusion criteria included the presence of any disease which could affect biochemical parameters and clinical symptoms. The study protocol was approved by the bioethics and Gülhane Training and Research Hospital Research Committee with the number of
19/156 on April 30th of 2019, and all procedures were performed in compliance with the Helsinki Declaration. All patients were informed about the study and written informed consent was obtained. In patients' routine controls, data were recorded about their nutrition by using food consumption recording form, biochemical parameters including serum hemoglobin, ferritin, vitamin $B_{12}$, thyroid stimulating hormone, calcium, magnesium, folic acid, vitamin D, total protein, albumin, sedimentation, CRP levels. Twenty-four hours dietary recall was taken by investigator to determine daily nutrient intake of patients. Portion sizes and volumes were estimated with a portion size picture book including 120 photographs of foods, each with 3-5 different portion sizes (10). The BeBiS software version 7.2 was used to calculate daily intake of energy, macro and micronutrients (11). Dietary intakes of patients were assessed according to the Turkey-Specific Nutrition Guide (12). Clinical symptoms were evaluated by using the Short form of MacGill Pain Questionnaire (SFMPQ), Fibromyalgia Impact Questionnaire (FIQ) and Pittsburg Sleep Quality Index (PSQI).

\section{Statistical Analysis}

Statistical analyses were performed with Statistical Package for Social Sciences (SPSS) version 22.0 for Mac (SPSS Inc., Chicago, IL). Continuous variables were presented as mean \pm standard deviation. Qualitative variables were presented as number and percentage. The Kolmogorov-Smirnov test was used to determine the normality of data distribution. Correlation analyses were done with the Pearson test. A value of $p<0.05$ was considered to be statistically significant.

\section{Results}

Main characteristics of the patients are given in Table 1. Most of the patients were female (90.1\%) and most of them were married $(82.7 \%)$. Education duration was generally low (1-5 years in $49.4 \%$ of the patients). Laboratory findings are given in Table 2. All parameters except 25-OH D were in normal range. The mean $25-\mathrm{OH}$ D level was $22.8 \pm 14.1 \mathrm{ng} / \mathrm{mL}$.

FIQ total, SFMPQ, and PSQI scores of the patients are given in Table 3. FIQ total score was $48.7 \pm 23.5$, SFMPQ total pain rating index was $12.9 \pm 8.6$, and PSQI-total was 9.7 \pm 4.5 .

The correlation analysis between serum vitamin $\mathrm{B}_{12}$ and clinical symptoms showed a weak negative correlation with FIQ ( $r=-0.257, p=0.021)$, sleep duration ( $r=-0.317, p=0.004)$, sleep disturbance $(r=-0.279, p=0.012)$, sleep quality $(r=-0.235$, $p=0.035)$, and PSQI total score $(r=-0.316, p=0.004)$. There was no correlation between other biochemical parameters and clinical symptoms.

The mean intakes of energy, carbohydrate, protein and fat were $1919.5 \pm 618.8 \mathrm{kcal}, 212.2 \pm 94.7 \mathrm{~g}, 71.9 \pm 21.2 \mathrm{~g}$, and $84.0 \pm 31.9 \mathrm{~g}$, respectively. When daily energy percentage from carbohydrate, protein and fat were assessed according 
to the Turkey-Specific Nutrition Guide, energy percentage from carbohydrate was over reference values in five $(6.17 \%)$ patients, energy percentage from protein was over reference values in $10(12.3 \%)$ patients and energy percentage from fat was over reference values in $60(74.1 \%)$ patients. In addition, the contribution of saturated fat to energy was determined above the recommended level in $69(85.2 \%)$ individuals. The correlation between FIQ, SFMPQ, macronutrients, and some micronutrients (which were significantly correlated) were shown in Table 4. Dietary energy, carbohydrate, protein, and percentage of energy from dietary protein were not correlated with FIQ and SFMPQ. The percentage of energy from dietary carbohydrate showed a moderate positive correlation with FIQ ( $r=0.383$, $p<0.001)$. Dietary fat showed moderate negative correlation with FIQ $(r=-0.29, p=0.007)$. While dietary saturated fat had a

\begin{tabular}{|lll|}
\hline \multicolumn{2}{l}{ Table 1. Main characteristics of patients } \\
\hline & Mean & SD \\
\hline Age (years) & 48.9 & 10.5 \\
\hline Gender & $\mathbf{n}$ & $\%$ \\
\hline Female & 73 & 90.1 \\
\hline Male & 8 & 9.9 \\
\hline Education & & \\
\hline Illiterate & 3 & 3.7 \\
\hline 1-5 years & 40 & 49.4 \\
\hline 6-10 years & 3 & 3.7 \\
\hline 10-15 years & 16 & 19.8 \\
\hline$>15$ years & 19 & 23.5 \\
\hline Marital status & & \\
\hline Married & 67 & 82.7 \\
\hline Single & 14 & 17.3 \\
\hline SD: Standard deviation & & \\
\hline
\end{tabular}

\begin{tabular}{|lll|}
\hline \multicolumn{3}{l}{ Table 2. Laboratory findings of patients } \\
\hline Parameter & Mean & SD \\
\hline Hemoglobin $(\mathrm{g} / \mathrm{dL})$ & 13.2 & 1.1 \\
\hline ESR $(\mathrm{mm} / \mathrm{h})$ & 15.7 & 10.6 \\
\hline CRP $(\mathrm{mg} / \mathrm{L})$ & 3.4 & 4.4 \\
\hline Vitamin $B_{12}(\mathrm{pg} / \mathrm{dL})$ & 327.8 & 205.7 \\
\hline Folic acid $(\mathrm{ng} / \mathrm{mL})$ & 8.3 & 3.8 \\
\hline Ferritin $(\mathrm{ng} / \mathrm{mL})$ & 31.7 & 30.1 \\
\hline Ca $(\mathrm{mg} / \mathrm{dL})$ & 9.3 & 0.9 \\
\hline Mg $(\mathrm{mg} / \mathrm{dL})$ & 2.1 & 0.7 \\
\hline 25-OH D $(\mathrm{ng} / \mathrm{mL})$ & 22.8 & 14.1 \\
\hline Total protein $(\mathrm{mg} / \mathrm{dL})$ & 7.1 & 0.8 \\
\hline Albumin $(\mathrm{mg} / \mathrm{dL})$ & 4.2 & 0.3 \\
\hline TSH $(\mathrm{mU} / \mathrm{L})$ & 2.2 & 1.7 \\
\hline ESR: Erythrocyte sedimentation rate, CRP: C-reactive protein, TSH: Thyroid \\
stimulating hormon, Ca: Calcium, Mg: Magnesium, SD: Standard deviation
\end{tabular}

moderate positive correlation with $\mathrm{FIQ}(\mathrm{r}=0.361, \mathrm{p}<0.001)$, dietary unsaturated fat had a weak negative correlation with FIQ ( $r=-$ $0.228, p=0.041)$. Percentage of energy from dietary fat showed a negative moderate correlation with FIQ $(r=-0.411, p<0.001)$ and a weak negative correlation with SFMPQ-visual analogue scale (VAS) subscore $(r=-0.225, p=0.044)$. Dietary fiber displayed a moderate negative correlation with $\mathrm{FIQ}(\mathrm{r}=-0.357, \mathrm{p}<0.001)$, SFMPQ-present pain intensity (PPI) $(r=-0.357, p<0.001)$, and SFMPQ-VAS ( $r=-0.419, p<0.001)$ subscores. In addition to dietary energy and above mentioned macronutrients, some vitamins and minerals (folic acid, calcium, phosphor, vitamin B1, vitamin B2, biotin, iron, zinc, and magnesium) showed a weak to moderate negative correlations with FIQ, SFMPQ-PPI, and SFMPQ-VAS subscores (as presented in Table 4).

\section{Discussion}

In this study of 81 patients who were diagnosed with fibromyalgia, results have shown some associations between biochemical parameters, dietary intake and clinical assessments of pain, sleep quality and current health status. The mean vitamin $B_{12}$ level was in normal range and vitamin $B_{12}$ levels showed a negative correlation with clinical scores such as FIQ and sleep scores. Although low vitamin $B_{12}$ levels were reported with pain related pathologies before (13), studies including patients with fibromyalgia demonstrated no link between serum vitamin $B_{12}$ levels and fibromyalgia $(14,15)$. In a study by Regland et al. (16), cerebrospinal fluid vitamin $B_{12}$ levels were correlated with fatigue and psychological ratings. This result was similar to the result in the current study notwithstanding sample differences. We suppose that more studies are necessary to firmly report a vitamin $B_{12}$ effect on fibromyalgia symptoms.

\begin{tabular}{lll}
$\begin{array}{l}\text { Table 3. Fibromyalgia Impact Questionnaire total, Short } \\
\text { form of MacGill Pain Questionnaire, and Pittsburg Sleep } \\
\text { Quality Index scores of the patients }\end{array}$ \\
\hline & Mean & SD \\
\hline FIQ total & 48.7 & 23.5 \\
\hline McGill sensory & 10.1 & 6.3 \\
\hline McGill affective & 2.9 & 3.1 \\
\hline Total pain rating index & 12.9 & 8.6 \\
\hline McGill present pain intensity & 3.1 & 1.0 \\
\hline McGill VAS & 5.2 & 2.3 \\
\hline PSQI & & \\
\hline Sleep duration & 1.1 & 1.0 \\
\hline Sleep latency & 1.8 & 1.0 \\
\hline Sleep disturbance & 1.9 & 0.8 \\
\hline Habitual sleep efficiency & 0.8 & 1.1 \\
\hline Sleep medication use & 0.2 & 0.7 \\
\hline Daytime dysfunction & 1.7 & 1.0 \\
\hline PSQI-total & 9.7 & 4.5 \\
\hline
\end{tabular}

FIQ: Fibromyalgia Impact Questionnaire, PSQI: Pittsburg Sleep Quality Index, VAS: Visual analogue scale, SD: Standard deviation 


\begin{tabular}{|c|c|c|c|c|c|c|c|c|c|c|c|c|}
\hline & \multicolumn{2}{|c|}{ FIQ-total } & \multicolumn{2}{|c|}{$\begin{array}{l}\text { McGill } \\
\text { sensory }\end{array}$} & \multicolumn{2}{|c|}{$\begin{array}{l}\text { McGill } \\
\text { affective }\end{array}$} & \multicolumn{2}{|c|}{$\begin{array}{l}\text { Total pain } \\
\text { rating index }\end{array}$} & \multicolumn{2}{|c|}{$\begin{array}{l}\text { McGill present } \\
\text { pain intensity }\end{array}$} & \multicolumn{2}{|c|}{ McGill VAS } \\
\hline & $r$ & p & $r$ & $\mathbf{p}$ & $r$ & p & $r$ & p & $r$ & p & $r$ & $p$ \\
\hline Energy & -0.072 & 0.524 & 0.003 & 0.981 & 0.033 & 0.769 & 0.014 & 0.902 & -0.026 & 0.815 & -0.021 & 0.854 \\
\hline Cho & 0.130 & 0.247 & 0.020 & 0.861 & -0.043 & 0.704 & -0.001 & 0.993 & 0.030 & 0.793 & 0.094 & 0.406 \\
\hline Cho \% & 0.383 & $<0.001$ & 0.084 & 0.457 & -0.103 & 0.358 & 0.054 & 0.829 & 0.123 & 0.276 & 0.203 & 0.070 \\
\hline Protein & -0.196 & 0.080 & 0.013 & 0.907 & 0.076 & 0.498 & 0.037 & 0.742 & -0.045 & 0.692 & -0.096 & 0.395 \\
\hline Protein \% & -0.130 & 0.249 & -0.042 & 0.710 & 0.034 & 0.766 & -0.019 & 0.868 & -0.012 & 0.918 & -0.050 & 0.658 \\
\hline Fat & -0.29 & 0.007 & -0.025 & 0.823 & 0.110 & 0.330 & 0.021 & 0.853 & -0.106 & 0.345 & -0.172 & 0.126 \\
\hline Fat $\%$ & -0.411 & $<0.001$ & -0.077 & 0.494 & 0.119 & 0.292 & -0.014 & 0.902 & -0.135 & 0.229 & -0.225 & 0.044 \\
\hline Saturated fat & 0.361 & $<0.001$ & 0.105 & 0.350 & -0.017 & 0.881 & -0.083 & 0.460 & -0.055 & 0.624 & -0.123 & 0.274 \\
\hline Unsaturated fat & -0.228 & 0.041 & 0.015 & 0.891 & 0.158 & 0.158 & 0.068 & 0.545 & -0.117 & 0.296 & -0.173 & 0.123 \\
\hline Cholesterol & -0.213 & 0.056 & -0.106 & -0.344 & 0.013 & 0.907 & -0.073 & 0.515 & -0.018 & 0.871 & -0.067 & 0.551 \\
\hline Fiber & -0.358 & $<0.001$ & 0.111 & 0.325 & 0.116 & 0.302 & 0.123 & 0.274 & -0.357 & $<0.001$ & -0.419 & $<0.001$ \\
\hline Vitamin B1 & -0.246 & 0.027 & 0.040 & 0.722 & 0.027 & 0.811 & 0.039 & 0.729 & -0.274 & 0.013 & -0.320 & $<0.001$ \\
\hline Vitamin B2 & -0.393 & $<0.001$ & -0.221 & 0.047 & -0.083 & 0.464 & -0.192 & 0.086 & -0.265 & 0.017 & -0.356 & $<0.001$ \\
\hline Biotin & -0.262 & 0.018 & -0.094 & 0.402 & -0.053 & 0.641 & -0.088 & 0.434 & -0.315 & 0.004 & -0.384 & $<0.001$ \\
\hline Folic acid & -0.405 & $<0.001$ & 0.003 & 0.979 & 0.100 & 0.374 & 0.038 & 0.735 & -0.276 & 0.013 & -0.498 & $<0.001$ \\
\hline Vitamin C & -0.191 & 0.088 & 0.017 & 0.881 & 0.232 & 0.037 & 0.096 & 0.395 & -0.118 & 0.293 & -0.287 & 0.009 \\
\hline Calcium & -0.473 & $<0.001$ & -0.237 & 0.034 & -0.124 & 0.269 & -0.218 & 0.05 & -0.274 & 0.013 & -0.418 & $<0.001$ \\
\hline Magnesium & -0.259 & 0.020 & 0.022 & 0.847 & 0.044 & 0.694 & 0.032 & 0.777 & -0.227 & 0.042 & -0.335 & 0.002 \\
\hline Phosphor & -0.435 & $<0.001$ & -0.122 & 0.277 & -0.023 & 0.840 & -0.098 & 0.385 & -0.263 & 0.018 & -0.364 & 0.001 \\
\hline Iron & -0.323 & 0.003 & 0.081 & 0.472 & 0.084 & 0.453 & 0.090 & 0.425 & -0.271 & 0.014 & -0.390 & $<0.001$ \\
\hline Zinc & -0.273 & 0.014 & -0.030 & 0.791 & 0.036 & 0.752 & -0.009 & 0.935 & -0.184 & 0.099 & -0.245 & 0.028 \\
\hline
\end{tabular}

SFMPQ: Short form of MacGill Pain Questionnaire, FIQ: Fibromyalgia Impact Questionnaire, VAS: Visual analogue scale, Cho: Carbohydrate

Vitamin $D$ is also important in both inflammatory and pain pathways. We know an association between vitamin $D$ deficiency and fibromyalgia, but in fact, little is known about its mechanism in fibromyalgia. A recent review showed that patients with fibromyalgia had low levels of vitamin $D$ according to healthy controls (17). Literature shows contradictory results about the effect of vitamin $D$ on pain or symptom control, with no clear results indicating the role of supplementation in the management of fibromyalgia. Although there are studies that found negative correlation between pain or tender points count and vitamin $D$ levels, the remaining studies could not describe a correlation (18). In a study it was found that fibromyalgia patients with vitamin $D$ levels $\leq 20 \mathrm{ng} / \mathrm{mL}$ to be more likely to have humor disruption, confusion, memory deterioration, sleep problems, palpitations and restless-leg syndrome (19). In addition, vitamin D supplements are reported to improve disease symptoms in patients with fibromyalgia $(20,21)$. In the current study, the mean vitamin D level was $22.8 \pm 14.1 \mathrm{ng} / \mathrm{mL}$ and thirty patients showed vitamin D insufficiency. However, there was no correlation between serum vitamin $D$ levels and clinical symptoms.
The hypothesis about connection between dietary patterns and fibromyalgia symptoms has directed clinicians to investigate the effects of different diets on fibromyalgia symptoms (8). Raw vegetarian diet, low FODMAPs diet, hypocaloric diet, monosodium glutamate- and aspartame-free diet are some of the dietary patterns which have been studied to date and results on pain, quality of life and sleep are contradictory. A study showed that a pure vegetarian, raw diet provided significant improvements in fibromyalgia symptoms (pain, physical performance, function and quality of life) $(22,23)$. Another study reported that after a three-months vegan diet, there were significant improvements in pain scores, health assessment questionnaire scores, general health questionnaire, quality of sleep and morning stiffness (24). In a randomized controlled trial, it was shown that lacto-vegetarian diet combined with core stabilization exercises in patients with fibromyalgia who had low back pain provided pain reduction and body composition improvement (25). In another study, it was shown that a glutenfree diet improved all symptoms including pain, tender points, function, gastrointestinal complaints and fatigue (26). Despite the fact that there are studies in the literature about diet- 
symptom association, few studies investigated macronutrient and micronutrient content of the diet. In the current study, we found dietary energy percent from carbohydrate, fat, and fiber association with FIQ and SFMPQ.

Fibromyalgia and obesity are very closely associated pathologies and obesity and carbohydrate-rich diets are potential factors that may affect the severity of symptoms, pain, and disease activity in fibromyalgia syndrome $(27,28)$. We also found similar results in the current study. The percent of energy from carbohydrate showed a moderate positive correlation with FIQ. To date, some investigators tended to enlighten the possible relation between glucose metabolism and pain, but there is no significant result yet (29). In the current study, interestingly the percent of energy from fat showed a moderate negative correlation with FIQ and dietary fat showed a moderate negative correlation with FIQ. However, when we reanalyzed fat content considering saturated and unsaturated fat, saturated fat showed a moderate positive correlation while unsaturated fat showed a weak negative correlation with FIQ. We suggest that, according to this result, saturated fat content of diet may be the factor which worsens symptoms of the patient. Intake of saturated fat is increasing day by day due to processed food and fast food consumption. Diets containing excessive saturated fat are suspected to cause chronic diseases due to chronic low degree inflammation (30-33).

Plant-enriched diets are reported to increase nitric oxide levels which may help to relieve pain in fibromyalgia patients (8). In the current study, the amount of dietary fiber showed a moderate negative correlation with FIQ, SFMPQ-VAS and SFMPQ-PPI. Different from other dietary content, fiber showed a correlation with SFMPQ-VAS and SFMPQ-PPI and this gives a rise to the thought that increasing fiber content of diet may help relieving pain.

Redox balance changes in cells due to nutritional deficiencies like iron, magnesium, iodine, melatonin, zinc, selenium and branched chain amino acids can have a potential role in fibromyalgia symptoms (34). Although magnesium and selenium are suspected ions in physiopathology and symptom severity of fibromyalgia, a review showed that magnesium and malic acid use did not significantly change pain ratings or depressive symptoms in patients with fibromyalgia (35). While some authors reported that serum levels of selenium and magnesium were not significantly different from controls (36), another study showed a decrease in magnesium and zinc levels, but no significant difference in selenium levels. Also, serum magnesium and zinc levels were associated with clinical parameters, indicating a possible role of these two elements in fibromyalgia etiopathogenesis (37). However, this study did not investigate nutritional deficiency or low magnesium or zinc dietary intake. In the current study, we evaluated dietary intake of magnesium and found that dietary magnesium showed a weak negative correlation with FIQ and SFMPQ-VAS. As it can be realized from the above mentioned results, cross-sectional or interventional studies show contradictory results.

In addition to study methodology, included patients in the current study were given nutrition advice according to dietary recalls. To the best of our knowledge, the current study is the first on the relationship between nutrition, biochemical parameters and clinical symptoms in fibromyalgia patients. The cross-sectional design can be interpreted as the limitation of this study. There are also strengths of the study. Large sample size and comprehensive assessment of patients with multiple questionnaires are the strengths of the study. Well-designed clinical trials about the effect of the dietary interventions on fibromyalgia patients are necessary in order to understand more details about the potential benefits from nutrition.

\section{Conclusion}

The current study results suggest that a healthy diet habit consisting of low saturated fat, carbohydrates and higher fiber, vitamins and minerals (folic acid, calcium, phosphor, vitamin $B 1$, vitamin $B 2$, vitamin $B_{12}$, biotin, iron, magnesium, zinc) may provide an improvement in fibromyalgia symptoms. We also suggest that dietary assessment and interventions should be a part of the management of patients with fibromyalgia.

\section{Ethics}

Ethics Committee Approval: The study protocol was approved by the bioethics and Gülhane Training and Research Hospital Research Committee with the number of 19/156 on April $30^{\text {th }}$ of 2019 , and all procedures were performed in compliance with the Helsinki Declaration.

Informed Consent: All patients were informed about the study and written informed consent was obtained.

Peer-review: Internally peer-reviewed.

\section{Authorship Contributions}

Surgical and Medical Practices: Ö.K., K.T.A., Concept: Ö.K., K.T.A., Design: Ö.K., K.T.A., Data Collection or Processing: Ö.K., K.T.A., Analysis or Interpretation: Ö.K., K.T.A., Literature Search: Ö.K., K.T.A., Writing: Ö.K., K.T.A.

Conflict of Interest: No conflict of interest was declared by the authors.

Financial Disclosure: The authors declared that this study received no financial support

\section{References}

1. Wallace DJ, Hallegua DS. Fibromyalgia: The gastrointestinal link. Curr Pain Headache Rep. 2004;8:364-368.

2. Romano GF, Tomassi S, Russell A, Mondelli V, Pariante $\mathrm{CM}$. Fibromyalgia and Chronic Fatigue: The Underlying 
Biology and Related Theoretical Issues. Adv Psychosom Med. 2015;34:61-77.

3. Arranz LI, Canela MÁ, Rafecas M. Dietary aspects in fibromyalgia patients: results of a survey on food awareness, allergies, and nutritional supplementation. Rheumatol Int. 2012;32:2615-2621.

4. Clauw DJ. Fibromyalgia and related conditions. Mayo Clin Proc. 2015;90:680-692.

5. Bazzichi L, Rossi A, Massimetti G, et al. Cytokine patterns in fibromyalgia and their correlation with clinical manifestations. Clin Exp Rheumatol. 2007;25:225-230.

6. Carding S, Verbeke K, Vipond DT, Corfe BM, Owen LJ. Dysbiosis of the gut microbiota in disease. Microb Ecol Health Dis. 2015;26:26191.

7. Marum AP, Moreira C, Tomas-Carus P, Saraiva F, Guerreiro CS. A low fermentable oligo-di-mono-saccharides and polyols (FODMAP) diet is a balanced therapy for fibromyalgia with nutritional and symptomatic benefits. Nutr Hosp. 2017;34:667-674.

8. Bjørklund G, Dadar M, Chirumbolo S, Aaseth J. Fibromyalgia and nutrition: Therapeutic possibilities? Biomed Pharmacother. 2018;103:531-538.

9. Ortancil O, Sanli A, Eryuksel R, Basaran A, Ankarali H. Association between serum ferritin level and fibromyalgia syndrome. Eur J Clin Nutr. 2010;64:308-312.

10. Rakıcıoğlu N, Tek Acar N, Ayaz A, Pekcan G. Yemek ve Besin Fotograf Kataloğu-Ölçü ve Miktarlar. 2nd ed. Ankara: Ata Ofset Matbaacılık; 2012.

11. Bebis Nutrition Data Base Software Data Base, 2004. The German Food Code and Nutrient Data Base (BLS II.3, 1999) with additions from USDA-sr and other sources, Istanbul, Turkey.

12. T.C. Sağlık Bakanlığı. Türkiye Beslenme Rehberi TÜBER 2015. Yayın No: 1031, Ankara 2016." Last accessed date: 2020 Feb 3. Available from: https://dosyasb.saglik. gov.tr/Eklenti/10915,tuber-turkiye-beslenme-rehberipdf. pdf

13. Zhang M, Han W, Hu S, Xu H. Methylcobalamin: a potential vitamin of pain killer. Neural Plast. 2013:2013;1-6.

14. De Carvalho JF, Silva DNF. Serum levels of vitamin B12 (cobalamin) in fibromyalgia. Rheumatology International. 2016;36:741-742.

15. Bengtsson $A$, Henriksson $K G$, Jorfeldt $L$, Kågedal $B$, Lennmarken C, Lindstrom F. Primary fibromyalgia. A clinical and laboratory study of 55 patients. Scand J Rheumatol. 1986;15:340-347.

16. Regland B, Andersson M, Abrahamsson L, Bagby J, Dyrehag LE, Gottfries CG. Increased concentrations of homocysteine in the cerebrospinal fluid in patients with fibromyalgia and chronic fatigue syndrome. Scand J Rheumatol. 1997;26:301-307.

17. Makrani AH, Afshari M, Ghajar M, Forooghi Z, Moosazadeh M. Vitamin D and fibromyalgia: a meta-analysis. Korean J Pain. 2017;30:250-257.
18. Ellis SD, Kelly ST, Shurlock JH, Hepburn ALN. The role of vitamin $D$ testing and replacement in fibromyalgia: a systematic literature review. BMC Rheumatol. 2018;2:28.

19. Olama SM, Senna MK, Elarman MM, Elhawary G. Serum vitamin $\mathrm{D}$ level and bone mineral density in premenopausal Egyptian women with fibromyalgia. Rheumatol Int. 2013;33:185-192.

20. De Carvalho JF, da Rocha Araújo FAG, da Mota LMA, Aires $\mathrm{RB}$, de Araujo RP. Vitamin D Supplementation Seems to Improve Fibromyalgia Symptoms: Preliminary Results. Isr Med Assoc J. 2018;20:379-381.

21. Mirzaei A, Zabihiyeganeh M, Jahed SA, Khiabani E, Nojomi $M$, Ghaffari S. Effects of vitamin D optimization on quality of life of patients with fibromyalgia: A randomized controlled trial. Med J Islam Repub Iran. 2018;32:29.

22. Donaldson MS, Speight N, Loomis S. Fibromyalgia syndrome improved using a mostly raw vegetarian diet: an observational study. BMC Complement Altern Med. $2001 ; 1: 7$.

23. Høstmark AT, Lystad E, Vellar OD, Hovi K, Berg JE. Reduced plasma fibrinogen, serum peroxides, lipids, and apolipoproteins after a 3-week vegetarian diet. Plant Foods Hum Nutr. 1993;43:55-61.

24. Kaartinen K, Lammi K, Hypen M, Nenonen M, Hanninen O, Rauma AL. Vegan diet alleviates fibromyalgia symptoms. Scand J Rheumatol. 2000;29:308-313.

25. Martínez-Rodríguez A, Leyva-Vela B, Martínez-García A, Nadal-Nicolás Y. Effects of lacto-vegetarian diet and stabilization core exercises on body composition and pain in women with fibromyalgia: randomized controlled trial. Nutr Hosp. 2018;35:392-399.

26. Rodrigo L, Blanco I, Bobes J, de Serres FJ. Effect of one year of a gluten-free diet on the clinical evolution of irritable bowel syndrome plus fibromyalgia in patients with associated lymphocytic enteritis: a case-control study. Arthritis Res Ther. 2014;16:421.

27. Kaleth AS, Slaven JE, Ang DC. Obesity moderates the effects of motivational interviewing treatment outcomes in fibromyalgia. Clin J Pain. 2018;34:76-81.

28. Timmerman GM, Calfa NA, Stuifbergen AK. Correlates of body mass index in women with fibromyalgia. Orthop Nurs. 2013;32:113-119.

29. de Sá Ribeiro GAC, Scola RH, Piovesan EJ, et al. The importance of lactic acid in migraines and fibromyalgia. Rev Bras Rheumatol. 2015;55: 471-476.

30. DiNicolantonio JJ, Lucan SC, O'Keefe JH. The evidence for saturated fat and for sugar related to coronary heart disease. Prog Cardiovasc Dis. 2016;58:464-472.

31. Nishida K, Otsu K. Inflammation and metabolic cardiomyopathy. Cardiovasc Res. 2017;113:389-398.

32. Kuzma JN, Cromer G, Hagman DK, et al. No differential effect of beverages sweetened with fructose, high fructose corn syrup, or glucose on systemic or adipose tissue inflammation in normal-weight to obese adults: a 
randomized controlled trial. Am J Clin Nutr. 2016;104:306314.

33. Ekici M, Kisa U, Arikan Durmaz S, Ugur E, Nergiz Unal R. Fatty acid transport receptor soluble CD36 and dietary fatty acid pattern in type 2 diabetic patients: a comparative study. Br J Nutr. 2018;119:153-162.

34. Arranz LI, Canela MA, Rafecas M. Fibromyalgia and nutrition, what do we know? Rheumatol Int. 2010;30:14171427.
35. Ferreira I, Ortigoza Á, Moore P. Magnesium and malic acid supplement for fibromyalgia. Medwave. 2019;19:e7633.

36. Eisinger J, Plantamura A, Marie PA, Ayavou T. Selenium and magnesium status in fibromyalgia. Magnes Res. 1994; 7:285-288.

37. Sendur OF, Tastaban E, Turan Y, Ulman C. The relationship between serum trace element levels and clinical parameters in patients with fibromyalgia. Rheumatol Int. 2008;28:11171121. 